\title{
Selective Advantage of Diffusing Faster
}

\author{
Simone Pigolotti ${ }^{1}$ and Roberto Benzi ${ }^{2}$ \\ ${ }^{1}$ Departament de Fisica i Enginyeria Nuclear, Universitat Politecnica de Catalunya Edifici GAIA, \\ Rambla Sant Nebridi 22, 08222 Terrassa, Barcelona, Spain \\ ${ }^{2}$ Dipartimento di Fisica, Universita' di Roma "Tor Vergata” and INFN, via della Ricerca Scientifica 1, 00133 Roma, Italy
}

(Received 18 July 2013; revised manuscript received 28 March 2014; published 9 May 2014)

\begin{abstract}
We study a stochastic spatial model of biological competition in which two species have the same birth and death rates, but different diffusion constants. In the absence of this difference, the model can be considered as an off-lattice version of the voter model and presents similar coarsening properties. We show that even a relative difference in diffusivity on the order of a few percent may lead to a strong bias in the coarsening process favoring the more agile species. We theoretically quantify this selective advantage and present analytical formulas for the average growth of the fastest species and its fixation probability.
\end{abstract}

Different physical, social, and biological systems can be described by models belonging to the voter model (VM) universality class [1]. An important example in biology is the neutral stepping stone model $[2,3]$ whose dynamics explains qualitative and quantitatively spreading and the fixation of competing populations on a Petri dish [4,5]. In statistical physics, the VM is characterized by the existence of two symmetric absorbing states and a coarsening process without surface tension. Its macroscopic dynamics corresponds to the Langevin equation

$$
\partial_{t} f(\mathbf{x}, t)=D \Delta f+\sqrt{\Gamma f(1-f)} \eta(\mathbf{x}, t),
$$

where $D$ is the diffusion constant, $\Gamma$ the noise amplitude, and $\eta(\mathbf{x}, t)$ is a $\delta$-correlated white noise. In biological applications, the field $f$ usually represents the frequency of an allele, i.e., the local fraction of individuals carrying a given mutation. When mutants have a "selective advantage" $s$ over the wild type (i.e., a difference in reproduction rate), an additional term appears in Eq. (1), which becomes a stochastic version of the celebrated Fisher-KolmogorovPetrovskii-Piscounov (FKPP) equation

$$
\partial_{t} f(\mathbf{x}, t)=D \Delta f+s f(1-f)+\sqrt{\Gamma f(1-f)} \eta(\mathbf{x}, t) .
$$

In the absence of noise, Eq. (2) predicts a well-defined range expansion velocity of the mutants, $v=2 \sqrt{D s}[6,7]$. The same result is valid in the presence of weak multiplicative noise, up to logarithmic corrections [8,9], while in the strong noise regime one should expect a difference expression for the velocity $[10,11]$.

The stochastic FKPP equation is radically different from the VM. For $s>0$, Eq. (2) predicts $f=1$ to be the deterministic asymptotic stable equilibrium of the system, the state $f=0$ being unstable. One can think of $s$ in a similar way as the external field in the Ising model, breaking the $f \leftrightarrow(1-f)$ symmetry and thus driving the system away from the critical point, which is recovered for $s=0$. Often, the critical behaviors of the Langevin equation such as variants of Eq. (1) can be understood by analyzing their corresponding deterministic dynamics, either by means of its associated mean field potential [12] or by the Hamiltonian dynamics obtained by the path integral formulations [13].

Because of the relevance of the VM in nonequilibrium phenomena, it is interesting to understand whether there exist more general, possibly nondeterministic mechanisms to break the VM universality class. In biological terms, this amounts to asking whether an effective selective advantage can be achieved without any asymmetry in the birth and death rates. For example, it has been recently shown [14] that an asymmetry in the carrying capacity (i.e., the global biological mass) of the two alleles can induce an effective selective advantage.

In this Letter, we show that an effective selective advantage emerges in a competition model between two species diffusing at different speeds, but otherwise neutral. In biology, this setting is relevant to assess the evolutionary importance of movement, for example, in species which exist in motile and nonmotile variants, such as bacteria with and without flagellum. We will show that, in this case, competition is biased towards the fastest species. This is equivalent to an effective selective advantage that depends both on noise and spatial fluctuations, and is proportional to both the noise amplitude and the difference in diffusivity.

We consider a model in which particles belonging to two different species $A$ and $B$ diffuse in space, reproduce according to the reactions $A \rightarrow 2 A$ and $B \rightarrow 2 B$, and die in a density-dependent fashion $(A+A / B \rightarrow A$ and $B+A / B \rightarrow A$ ) as result of competition. For simplicity, we assume all reactions occur at the same rate $\mu=1$. The system is a hypercube of size $L^{d}$ in $d$ dimensions with periodic boundary conditions (see $[15,16]$ for details on the implementation). We call $D+\delta D$ and $D$ the diffusion 
constants of species $A$ and $B$ respectively. When $\delta D=0$, the dynamics of the model is characterized by a coarsening process, as shown in the two-dimensional example of Fig. (1), left column. We will later argue that this coarsening belongs to the universality class of the VM. Instead, Fig. (1), right column shows that a small difference in the diffusivity of the two species, $\delta D / D=5 \%$ in this case, imposes a non-negligible bias on the coarsening dynamics and, in particular, confers an advantage to the species having a larger diffusivity. A similar behavior can be observed also in 1D simulations.

We derived macroscopic equations for the concentrations of the two species $c_{A}(\mathbf{x}, t)$ and $c_{B}(\mathbf{x}, t)$ [15-18]. The result is

$\partial_{t} c_{A}=(D+\delta D) \nabla^{2} c_{A}+\mu c_{A}\left(1-c_{A}-c_{B}\right)+\sigma_{A} \xi_{A}(\mathbf{x}, t)$,

$\partial_{t} c_{B}=D \nabla^{2} c_{B}+\mu c_{B}\left(1-c_{A}-c_{B}\right)+\sigma_{B} \xi_{B}(\mathbf{x}, t)$,

where $\sigma_{A}=\sqrt{\mu c_{A}\left(1+c_{A}+c_{B}\right) / N}$ and, similarly for $\sigma_{B}$. The parameter $\mu / N$ is the genetic drift and $N$ can be
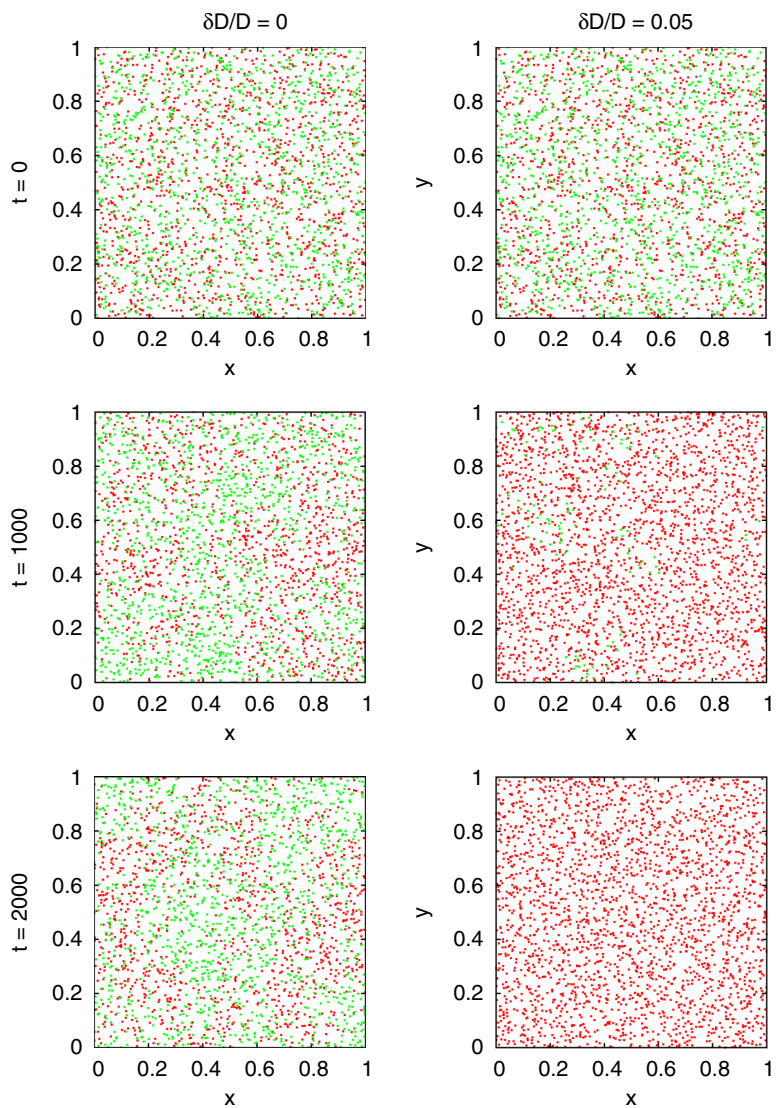

FIG. 1 (color online). Snapshots of 2D configurations of the particle model at different times. In all panels, parameters are $N=10^{4}$ and $D=10^{-4}$. Details of the particle simulations are in [16]. (Left column) The two species have the same diffusivity. (Right column) The red species has diffusivity $D+\delta D$ with $\delta D / D=0.05$. To help the viewer, configurations have been down sampled (one every four particles, chosen at random, is shown). interpreted as the density of particles corresponding to a macroscopic concentration $c=1 . \xi_{A}(\mathbf{x}, t)$ and $\xi_{B}(\mathbf{x}, t)$ are independent delta-correlated (in space and time) noise sources, $\quad\left\langle\xi_{i}(\mathbf{x}, t) \xi_{j}\left(\mathbf{x} \prime, t^{\prime}\right)\right\rangle=\delta_{i j} \delta(\mathbf{x}-\mathbf{x} \prime) \delta\left(t-t^{\prime}\right) . \quad$ An equation for the relative concentration of one species $f \equiv$ $c_{A} /\left(c_{A}+c_{B}\right)$ can be obtained from Eqs. (3) by means of Ito's formula $[15,18]$. Performing the calculation and neglecting fluctuations of the total particle density by imposing $c_{A}+c_{B}=1$ at the end of the procedure yields

$$
\partial_{t} f(\mathbf{x}, t)=D \nabla^{2} f+\delta D(1-f) \nabla^{2} f+\sigma \xi(\mathbf{x}, t),
$$

where $\sigma=\sqrt{2 \mu f(1-f) / N}$. Equation (4) constitutes the starting point of our analysis. We remark that, while we neglected fluctuations of the total density $c_{A}+c_{B}$, the fact that the total density is not strictly conserved is crucial to derive Eq. (4). This corresponds to the fact that it is impossible to have two species with different diffusion constants in a lattice model: if each site is strictly occupied by one spin, then the effective diffusivity of the two species is equal by constraint. Setting $\delta D=0$ in (4) one retrieves Eq. (1) describing the VM universality class $[12,15,19]$. Although (4) has been derived thinking of the continuum limit of a biological model, we argue that its validity is more general, as the term proportional to $\delta D$ is the simplest, nontrivial way to account for a difference in diffusivity between the two species. In the following, we will study how this term affects the dynamics by breaking the VM universality class.

We start by focusing on the 1D case and study the time evolution of the integrated mean concentration $F(t)=\langle f\rangle$, where $\langle.$.$\rangle denotes an average over space and noise. From$ Eq. (4) we obtain

$$
\frac{d F}{d t}=\delta D\left\langle(\nabla f)^{2}\right\rangle>0
$$

The above equation already shows that $F(t)$ is a growing function of time for any $\delta D>0$. The behavior of $F(t)$ is presented in Fig. 2 in 1D simulations of the particle model, starting with uniformly distributed populations but a more abundant slow species, so that $F(0)=0.1$. Notice how $F(t)$ decreases at increasing $N$ and increasing $D$ at constant $\delta D / D$. A straightforward calculation [16] shows that

$$
\frac{d F(t)}{d t}=\left.\frac{\delta D}{2} \nabla^{2} H(x, t)\right|_{x=0},
$$

where we introduced the two point connected correlation function (heterozygosity in biological language) $H(x=$ $\left.x_{1}-x_{2}, t\right)=\left\langle f\left(x_{1}\right)\left[1-f\left(x_{2}\right)\right]+f\left(x_{2}\right)\left[1-f\left(x_{1}\right)\right]\right\rangle$, which is function of $x_{1}-x_{2}$, only due to translational invariance. For $\delta D=0$ the function $H(x, t)$ is explicitly known [19]. For $\delta D / D \ll 1$, we can use this result to evaluate the righthand side of Eq. (6) at first order in perturbation theory, 
i.e., by replacing the average $\langle\ldots\rangle$ with the average $\langle\ldots\rangle_{0}$ over the solvable case of $\delta D=0$ [16]. The result is

$$
\frac{d F(t)}{d t}=\frac{\delta D}{4 D \sqrt{\pi \varepsilon t_{f}}} H(0) G\left(t / t_{f}\right)
$$

where $G(x)=\exp (x) \operatorname{erfc}(\sqrt{x}), t_{f}=2 D N^{2}$ and the parameter $\epsilon$ is an ultraviolet cutoff that can be assumed to be of order 1. Using expression (7), we can cast the growth of $\delta F(t)=F(t)-F(0)$ into the scaling form

$$
\delta F(t)=H(0) \sqrt{t_{f}} \frac{\delta D}{D} \phi\left(t / t_{f}\right)
$$

where the scaling function $\phi$ does not depend on parameters and is $\phi(x) \sim x$ for small $x$. A collapse of curves for different values of $N$ and $D$ according to (8), presented in the inset of Fig. (2), supports our theory within statistical fluctuations. Notice that, at this order in perturbation theory, the presence of absorbing states is not predicted by Eq. (7). This means that the perturbative approach is expected to describe properly the dynamics only on times shorter than the global fixation time, i.e., $L^{2} / D$.

It is of interest to compare a difference in diffusivity to a selective advantage caused by a difference $s$ in reproduction rates, i.e., in the case of Eq. (2). Assuming $s \ll 1$ and averaging directly such term, one obtains that $F(t)$ evolves in this case according to $d F / d t=s H(0) G\left(t / t_{f}\right) / 2$. Comparing the latter expression with Eq. (7), it is natural to define an effective advantage given by

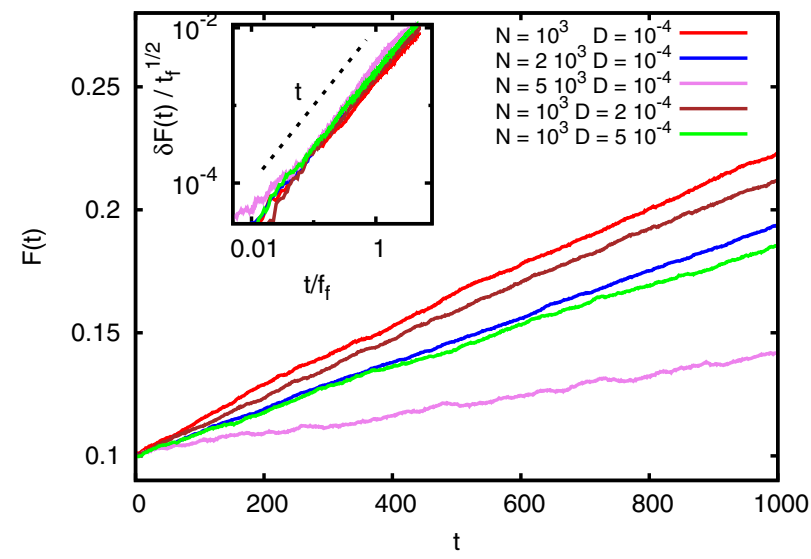

FIG. 2 (color online). Behavior of the average concentration of the fast species $F(t)$ at varying the number density $N$ and the diffusion constant of the slowest species $D$ in one dimension. In all simulations, the relative difference of diffusion constants is $\delta D / D=0.1$, the initial fraction of the fast species is $F(0)=0.1$, and the system size is $L=10$. Curves are averaged over $10^{3}$ realizations. The inset shows a data collapse according to Eq. (8). Linear scaling (black dashed line) is shown for comparison.

$$
s_{\mathrm{eff}}=\frac{\delta D}{2 D \sqrt{\epsilon \pi t_{f}}} .
$$

To tackle the problem in higher dimensions, let us start from the general evolution equation for the two point connected correlation function as obtained from Eq. (4) for $\delta D=0$ :

$$
\partial_{t} H(x, t)=2 D \nabla^{2} H-\frac{2 \mu}{N} H(0, t) \delta(x) .
$$

Because of the spatial regularization [10], the delta function resulting from Ito calculus must be interpreted as $\delta(x) \sim 1 / a^{d}$, where $a \sim \sqrt{2 D \epsilon}$ is the lattice spacing of the discrete stepping stone model. In an adiabatic approximation of Eq. (10), $\left.\nabla^{2} H\right|_{x=0}$ can be estimated as

$$
\left.\nabla^{2} H\right|_{x=0} \sim \frac{\mu H(0, t)}{D N(D \epsilon)^{d / 2}},
$$

which is consistent with the scaling of Eq. (8) for $d=1$. Evaluating Eq. (11) in $d=2$ yields $\left.\nabla^{2} H\right|_{x=0} \sim$ $\mu H(0, t) /\left(N D^{2} \epsilon\right)$ : i.e., the effective advantage becomes larger by a factor $1 / \sqrt{D \epsilon}$ with respect to the onedimensional case.

The interpretation of Eqs. (9) and (11) is that, after averaging over noise and space, the effect of a different diffusivity is analogous to that of a selective advantage. We now discuss the consequences for the peculiar coarsening properties of the VM. In two dimensions, the dynamics of the VM is characterized by a slow coarsening process where the density of interface decays as $\log ^{-1}(t)$ (see, e.g., [1]). In the continuum off-lattice case, the analogous of the density of interface is the local heterozigosity $H(x=0, t)$. Figure 3 shows how $H(x=0, t)$ displays the expected logarithmic decay in our particle model. When either a selective advantage or a diffusivity difference is present, this behavior is observed up to a time $\bar{t}$ (either proportional to the selective advantage $s$ or the diffusivity difference $\delta D$ ) after which $H(0, t)$ decays exponentially. This shows how both terms have a similar effect in driving the dynamics away from the VM critical point.

The effective selective advantage introduced in Eq. (9) can be used to study the probability of fixation $P_{\text {fix }}$, defined as the probability of reaching the absorbing state $f=1$ of Eq. (4). The fixation probability in terms of a selective advantage is given by the formula $[10,15]$

$$
P_{\text {fix }}(s)=\frac{1-\exp }{1-\exp (-2 s N)} .
$$

Assuming the same formula to hold in the case of different diffusivities with $s_{\text {eff }}$ replacing $s$ leads to an interesting prediction: $P_{\text {fix }}$ should not depend on $N$ as $s_{\text {eff }} \propto N^{-1}$. In Fig. 4(a) we show $P_{\text {fix }}$ as a function of $\delta D / D$ for different values of $N$, confirming this prediction. The black line is 


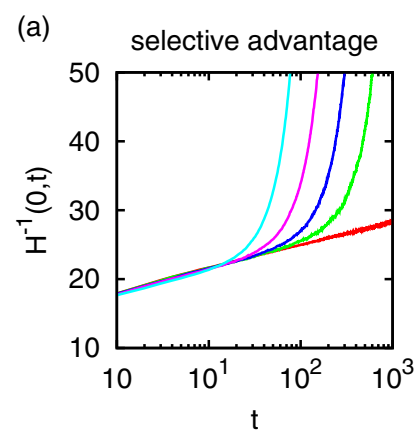

(b)

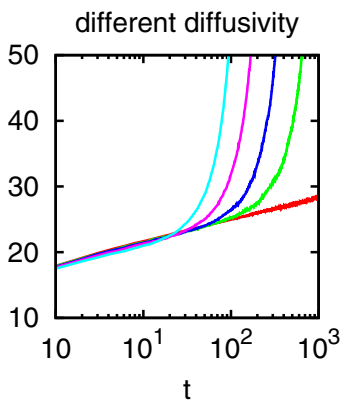

FIG. 3 (color online). Comparison of the coarsening dynamics in two dimensions in the presence of (a) selective advantage (i.e., faster reproduction rate of species $A$, and (b) difference in diffusivity. Parameters are $D=2 \times 10^{-5}$ and $N=4 \times 10^{4}$; the system is a square of side $L=1$. In (a), the selective advantage $s=0$ (red curve), $s=0.05,0.01,0.02,0.04$ (curves from right to left). In (b) we vary $\delta D / D=0$ (red curve), $\delta D / D=0.04,0.08$, $0.16,0.32$ (curves from right to left). In both panels, we plot the inverse density of interfaces $H^{-1}(x=0, t)$ as a function of time. Notice how the logarithmic behavior, characteristic of the VM is cut off on a time set by either the selective advantage or the difference in diffusivity.

the expression for $P_{\text {fix }}$, namely, obtained from $\epsilon \approx 0.5 \mu^{-1}$. This also confirms our initial assumption of $\epsilon \mu \sim 1$. Notice how the bias in the fixation probabilities shown in Fig. 4 is much stronger in two dimensions than in one dimension at equal parameter values, as predicted by Eq. (11)

We now briefly discuss the same problem in the presence of advection. Simulations in two dimensions shown in Fig. 4(b) show similar fixation probabilities with and without advection by an incompressible flow (details in [16]). In [16] we argue that in an incompressible flow Eq. (5) formally holds, leading to the same effective advantage for the species with larger diffusivity, as far as the typical scale of the turbulent scale is not too small.

To conclude, we have shown that a small difference in diffusivity can induce a breaking of the VM universality class with the critical parameter proportional to the noise amplitude. In the framework of population dynamics, this means that a difference in diffusivity between two species can bias the outcome of competition towards the more agile one. Notice that, while in the presence of a range expansion there exists an advantage for the fastest species which can be estimated by looking at the difference between the deterministic Fisher velocities $[5,20]$, the effect presented here is genuinely stochastic and constitutes a new example of a noise-induced advantage in population genetics $[14,21]$.

When considering a realistic biological scenario, the effective advantage given by a higher motility must be compared with its involved metabolic cost. In this respect, our result is reminiscent of a classic analysis of seed dispersal by Hamilton and May [22,23], which predicts an equilibrium, optimal level of dispersal even in a
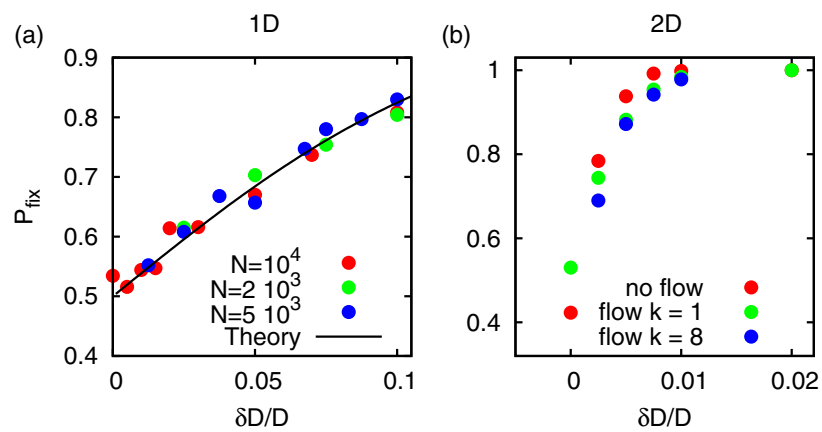

FIG. 4 (color online). (a) Fixation probabilities in one dimension. The black line is the theoretical prediction of Eq. (12). (b) Fixation probabilities in two dimensions, with and without fluid flow. In both panels we set $D=10^{-4}$.

homogeneous environment. Further, our preliminary results in the presence of fluid flows suggest that the same effect can be crucial also in turbulent marine environments. We expect our result to be also relevant in other fields where diffusion is known to affect crucially the dynamics, such as chemical kinetics [24], game theory [25], and synchronization [26]. Indeed, a term proportional to $(1-f) \nabla^{2} f$ would generally appear in the continuous description of systems characterized by two spatial concentrations diffusing at different speed, the proper dynamical evolution being described by Eq. (4).

We wish to thank M. Cencini, D. R. Nelson, and M. A. Muñoz for comments on the manuscript. S.P. acknowledges partial support from the Spanish research ministry through Grant No. FIS2012-37655-C02-01.

[1] I. Dornic, H. Chate, J. Chave, and H. Hinrichsen, Phys. Rev. Lett. 87, 045701 (2001).

[2] M. Kimura and G. H. Weiss, Genetics 49, 561 (1964).

[3] J. F. Crow and M. Kimura, An Introduction to Population Genetics (Blackburn Press, Caldwell, NJ, 2009).

[4] O. Hallatschek, P. Hersen, S. Ramanathan, and D. R. Nelson, Proc. Natl. Acad. Sci. U.S.A. 104, 19926 (2007).

[5] K. S. Korolev, M. J. Mueller, N. Karahan, A. W. Murray, O. Hallatschek, and D. R. Nelson, Phys. Biol. 9, 026008 (2012).

[6] R. A. Fisher, Ann. Eugenics 7, 353 (1937).

[7] A. Kolmogorov, N. Petrovsky, and N. Piscounov, Moscow Univ. Math. Bull. 1, 1 (1937).

[8] E. Brunet and B. Derrida, Phys. Rev. E 56, 2597 (1997).

[9] L. Pechenik and H. Levine, Phys. Rev. E 59, 3893 (1999).

[10] C. R. Doering, C. Mueller, and P. Smereka, Physica (Amsterdam) 325A, 243 (2003).

[11] O. Hallatschek and K. S. Korolev, Phys. Rev. Lett. 103, 108103 (2009).

[12] O. Al Hammal, H. Chate, I. Dornic, and M. A. Muñoz, Phys. Rev. Lett. 94, 23601 (2005).

[13] V. Elgart and A. Kamenev, Phys. Rev. E 74, 041101 (2006).

[14] O. Hallatschek, PLoS Comput. Biol. 7, e1002005 (2011). 
[15] S. Pigolotti, R. Benzi, P. Perlekar, M. H. Jensen, F. Toschi, and D. R. Nelson, Theor. Popul. Biol. 84, 72 (2013).

[16] See Supplemental Material at http://link.aps.org/supplemental/ 10.1103/PhysRevLett.112.188102 for mathematical demonstrations and an extended discussion on the possible role of advecting flows.

[17] C. Gardiner, Handbook of Stochastic Methods: For Physics, Chemistry and the Natural Sciences, Springer Series in Synergetics (Springer, New York, 2004).

[18] S. Pigolotti, R. Benzi, M. H. Jensen, and D. R. Nelson, Phys. Rev. Lett. 108, 128102 (2012).

[19] For a recent review, see K. Korolev, M. Avlund, O. Hallatschek, and D. R. Nelson, Rev. Mod. Phys. 82, 1691 (2010).
[20] O. Benichou, V. Calvez, N. Meunier, and R. Voituriez, Phys. Rev. E 86, 041908 (2012).

[21] E. Heinsalu, E. Hernandez-Garcia, and C. Lopez, Phys. Rev. Lett. 110, 258101 (2013).

[22] W. D. Hamilton and R. M. May, Nature (London) 269, 578 (1977).

[23] H. N. Comins, W. D. Hamilton, and R. M. May, J. Theor. Biol. 82, 205 (1980).

[24] P. L. Krapivsky, Phys. Rev. A 45, 1067 (1992).

[25] T. Reichenbach, M. Mobilia, and E. Frey, Nature (London) 448, 1046 (2007).

[26] N. Fujiwara, J. Kurths, and A. Diaz-Guilera, Phys. Rev. E 83, 025101 (2011). 